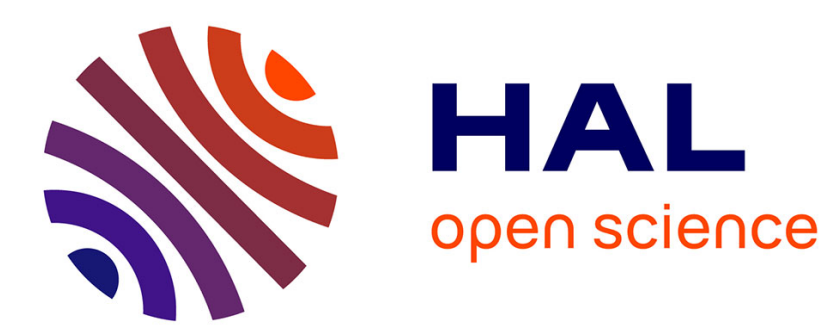

\title{
Particle dispersion in sheared suspensions: Crucial role of solid-solid contacts
}

Phong Pham, Bloen Metzger, Jason E. Butler

\section{To cite this version:}

Phong Pham, Bloen Metzger, Jason E. Butler. Particle dispersion in sheared suspensions: Crucial role of solid-solid contacts. Physics of Fluids, 2015, 27 (5), pp.051701. 10.1063/1.4919728 . hal-01442048

\section{HAL Id: hal-01442048 \\ https://hal.science/hal-01442048}

Submitted on 20 Jan 2017

HAL is a multi-disciplinary open access archive for the deposit and dissemination of scientific research documents, whether they are published or not. The documents may come from teaching and research institutions in France or abroad, or from public or private research centers.
L'archive ouverte pluridisciplinaire HAL, est destinée au dépôt et à la diffusion de documents scientifiques de niveau recherche, publiés ou non, émanant des établissements d'enseignement et de recherche français ou étrangers, des laboratoires publics ou privés. 


\section{AIP $\left.\right|_{\text {Fluids of }} ^{\text {Phyic }}$}

\section{Particle dispersion in sheared suspensions: Crucial role of solid-solid contacts}

Phong Pham, Bloen Metzger, and Jason E. Butler

Citation: Physics of Fluids (1994-present) 27, 051701 (2015); doi: 10.1063/1.4919728

View online: http://dx.doi.org/10.1063/1.4919728

View Table of Contents: http://scitation.aip.org/content/aip/journal/pof2/27/5?ver=pdfcov

Published by the AIP Publishing

\section{Articles you may be interested in}

Super-diffusion in sheared suspensions

Phys. Fluids 27, 041705 (2015); 10.1063/1.4918613

Topology of pair-sphere trajectories in finite inertia suspension shear flow and its effects on microstructure and rheology

Phys. Fluids 27, 043302 (2015); 10.1063/1.4917030

Effect of shear-induced diffusion on the transfer of heat across a sheared suspension

AIP Conf. Proc. 1542, 1071 (2013); 10.1063/1.4812120

Clouds of particles in a periodic shear flow

Phys. Fluids 24, 021703 (2012); 10.1063/1.3685537

Industrial application of ultrasound based in-line rheometry: From stationary to pulsating pipe flow of chocolate suspension in precrystallization process

Rev. Sci. Instrum. 75, 3164 (2004); 10.1063/1.1790585

\section{How to Simulate \&} Design Microfluidics

\section{Devices}

৩๐OMSOL
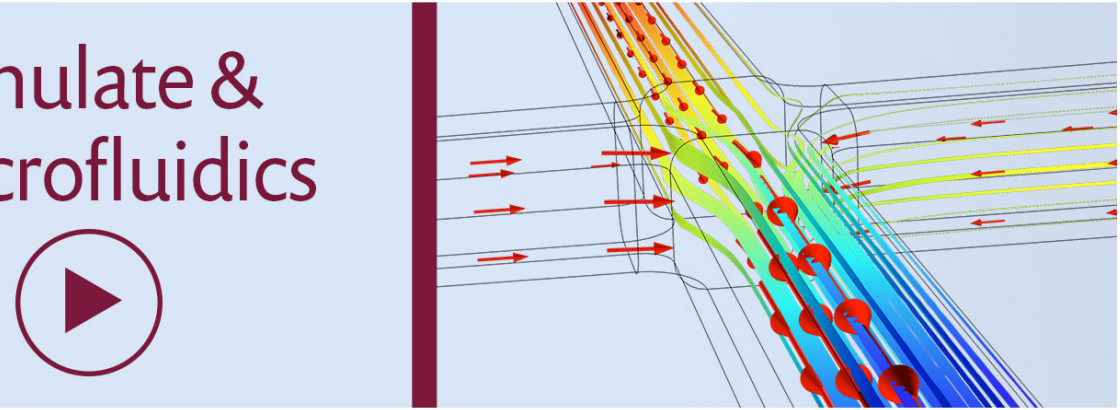


\title{
Particle dispersion in sheared suspensions: Crucial role of solid-solid contacts
}

\author{
Phong Pham, ${ }^{1,2}$ Bloen Metzger, ${ }^{1}$ and Jason E. Butler ${ }^{2}$ \\ ${ }^{1}$ IUSTI-CNRS UMR 7343, Aix-Marseille University, 13453 Marseille Cedex 13, France \\ ${ }^{2}$ Department of Chemical Engineering, University of Florida, Gainesville, \\ Florida 32611, USA
}

(Received 22 January 2015; accepted 20 April 2015; published online 1 May 2015)

\begin{abstract}
We performed high resolution measurements of the dynamics of non-Brownian and neutrally buoyant particles subjected to a periodic shear flow under low Reynolds number conditions. By changing the particle roughness and showing that it significantly affects the particle motion, we provide direct evidence that particle solidsolid contacts occur in viscous suspensions and strongly influence the particle dynamics. An accurate prediction of the particle trajectories is obtained with a minimal model that solely includes normal lubrication interactions and a frictionless contact force. () 2015 AIP Publishing LLC. [http://dx.doi.org/10.1063/1.4919728]
\end{abstract}

A different perspective, increasingly supported by experiments and numerical simulations, on how to describe sheared suspensions is emerging. Several experimental studies ${ }^{1-4}$ have suggested that solid-solid contact causes irreversible displacement of particles when colliding in viscous shear flows. Recent numerical studies demonstrated that in sheared suspensions, neither the long range hydrodynamic ${ }^{5}$ nor the lubrication interactions ${ }^{6}$ generate sufficient chaos to produce the irreversible behaviors observed experimentally; rather, contacts between particles were found to largely dominate the particle dispersion phenomenon. Clouds of particles, submitted to a periodic flow, progressively disperse until the volume fraction drops below a critical threshold where particles cease to fluctuate between cycles. ${ }^{7}$ This evolution also suggests that particle dispersion arises from contact interactions. More generally, simulations of shearing flows based on the simple rule that particles should not overlap ${ }^{8,9}$ generate results that compare well with experimental observations, yet these methods are exceedingly simple relative to simulation methods that incorporate the effects of multi-body hydrodynamics. ${ }^{10-12}$ Moreover, accurate measurements of pair distribution functions ${ }^{4}$ revealed a strong fore-aft asymmetry in which a "high concentration tail" forms in the recession quadrant. This distribution function was identified by Blanc et al. ${ }^{4}$ as consistent with that predicted for rough particles ${ }^{13}$ having a similar roughness, hence providing the most direct evidence yet that solid-solid contacts influence suspension dynamics.

All of these numerical and experimental observations support the idea that irreversible dynamics in sheared suspensions are dominated by particle contacts and their associated steric effects. This hypothesis is a promising one, since not having to account for the long range hydrodynamic interactions could greatly simplify calculations and would focus attention on what may be the essential ingredient to accurately model sheared suspensions. In this letter, we perform high resolution measurements of the dynamics of small numbers of particles submitted to a periodic flow. By altering the particle roughness and showing that this directly affects the particle motion, we provide evidence that particle solid-solid contacts occur in viscous suspensions and strongly influence the particle dynamics.

The experimental setup, shown in Figure 1(a), is an improved version of the one used by Metzger and Butler. ${ }^{7}$ A linear shear flow is generated by a transparent mylar belt that is mounted tightly on two rotating cylinders. The motion of the belt is controlled by a rotating stage (M-061.PD from PI Piezo-Nano Positioning) with high angular resolution $\left(3 \times 10^{-5} \mathrm{rad}\right)$. The cell was carefully designed to ensure reversibility upon flow reversal. To avoid unwanted secondary fluid re-circulations, this new configuration enables the fluid to flow under the cylinders and a constant spacing between the belt and the inner wall of the cell is maintained all around the cell. 


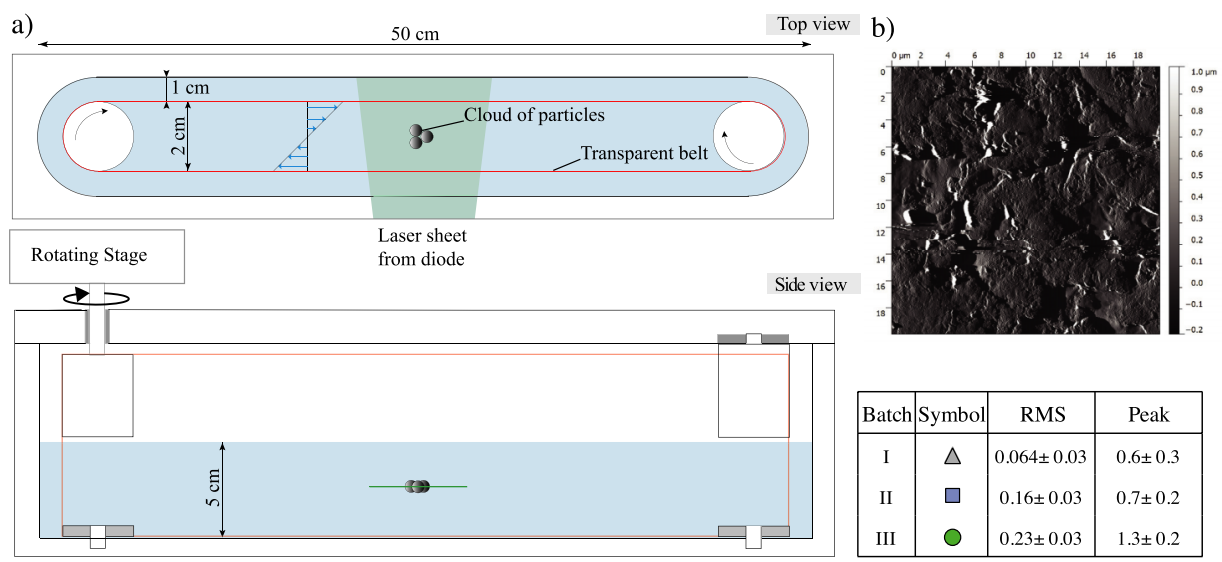

FIG. 1. (a) Sketch of the experimental shear-cell. A linear shear flow is generated by moving the transparent mylar belt (in red). Fluorescent particles, which are initially placed carefully in the shear plane, are illuminated with a thin laser sheet. (b) AFM image of a particle and table of the RMS and peak roughness in $\mu \mathrm{m}$, measured using AFM for the three different batches of particles.

The fluid is a mixture of Triton X-100 (76.241 wt. \%), zinc chloride (14.697 wt. \%), and water (9.062 wt. \%). It is a Newtonian fluid with a viscosity of $3 \mathrm{~Pa} \cdot \mathrm{s}$ and a density of $1.18 \mathrm{~g} / \mathrm{cm}^{3}$ at room temperature. The composition was chosen to match both the refractive index and density of the particles, which are mono-disperse spheres of diameter $d=2 \mathrm{~mm}$ that are made of poly-methylmethacrylate (PMMA). The roughness of the particles was modified by stirring the particles at $60 \mathrm{rpm}$ for one hour while immersed within a diamond paste solution (Presi Reflex LDP). Experiments were performed with three different batches of particles: the original ones and two other batches produced by polishing the original particles with solutions having different grades $(0.25 \mu \mathrm{m}$ and $15 \mu \mathrm{m})$. For each batch, the surface roughness of the particles was characterized by examining a total area of $400 \mu \mathrm{m}^{2}$ on four different particles. The data were processed using a standard method; ${ }^{14}$ an example result over a $20 \times 20 \mu \mathrm{m}$ area is shown in Figure 1(b) and the root mean square and peak values of the surface roughness are reported for each batch. The error values of roughness are reported in Figure 1(b) also.

Initially, the particles were carefully placed approximately $2.5 \mathrm{~cm}$ below the free surface of the fluid (see Fig. 1(a)) and were confirmed to be in the flow-gradient plane prior to starting an experiment. Visualization of the particles was enhanced by coating them with Rhodamine 6G. The coating was applied by soaking the particles in an equal parts solution of water and ethanol doped with Rhodamine $6 \mathrm{G}$ for $30 \mathrm{~min}$ at $60^{\circ} \mathrm{C}$. The particles were sheared forward and backward for 10 cycles at a strain amplitude of $\gamma_{0}=4$, where each cycle required $20 \mathrm{~s}$ to complete; the Reynolds number, based upon the particle diameter, is consequently $3 \times 10^{-4}$. A laser sheet, generated by a green laser diode ( $100 \mathrm{~mW}$ power and wavelength of $532 \mathrm{~nm}$ ), was used to illuminate the particles. The fluorescing particles were imaged from the top with a digital camera (Basler Ace) and a magnification lens (Sigma APO-Macro-180mm-F3.5-DG). A $550 \mathrm{~nm}$ high-pass filter was positioned before the lens to remove any light directly scattered by the particles. Images were then analyzed using a circular Hough transform to obtain the particle positions.

The experimental results were compared to a numerical model. This model represents a rudimentary description relative to the substantially more accurate models existing in the literature. ${ }^{10,13}$ However, it was built ${ }^{6}$ with the aim of determining the minimum physical ingredients needed to capture the dynamics of particles in sheared viscous suspensions. It includes the hydrodynamic drag on the particles from the background shear flow, a constant repulsive force, and the normal component of the lubrication force; long-range hydrodynamic interactions were not included in the model. In the absence of inertia, the sum of the hydrodynamic forces, $\mathbf{F}_{i}^{h}$, and the contact forces, $\mathbf{F}_{i}^{c}$, on each particle $i$ balances

$$
\mathbf{F}_{i}^{h}+\sum_{j \neq i}^{N} \mathbf{F}_{i j}^{c}=0
$$


In this minimal approach, we write the hydrodynamic forces as the sum of the drag due to the background shear flow and the normal components of the lubrication forces between neighboring particles,

$$
\mathbf{F}_{i}^{h}=3 \pi \mu d\left(\mathbf{u}_{i}-\mathbf{u}_{i \infty}\right)+\sum_{j(i)} \frac{3 \pi \mu d^{2}}{8 h_{i j}} \mathbf{n}_{i j}\left(\mathbf{u}_{i}-\mathbf{u}_{j}\right) \cdot \mathbf{n}_{i j},
$$

where $\mu$ denotes the fluid viscosity, $d$ the particle diameter, and $\mathbf{u}_{i}$ the velocity of particle $i$. The fluid velocity at $\mathbf{x}_{i}=\left(x_{i}, y_{i}\right)$, the position of particle $i$, is $\mathbf{u}_{i \infty}=\dot{\gamma} y_{i} \mathbf{e}_{x}$, where $\dot{\gamma}$ is the shear rate and $\mathbf{e}_{x}$ is the unit vector in the $x$-direction. Lubrication forces between particle $i$ and the particles $j(i)$ located within the lubrication range of particle $i$ depend upon the relative velocities and separation distance $h_{i j}=\left|\mathbf{x}_{i j}\right|-d$, where $\left|\mathbf{x}_{i j}\right|=\left|\mathbf{x}_{i}-\mathbf{x}_{j}\right|$, of each pair. The particles $j(i)$ located within the lubrication range of particle $i$ satisfy $2 \epsilon \leqslant h_{i j} \leqslant d / 2$, where $\epsilon$ denotes the dimensionless roughness of the particles. The algorithm only accounts for the normal component of lubrication, ${ }^{15}$ which acts along the vector $\mathbf{n}_{i j}=\mathbf{x}_{i j} /\left|\mathbf{x}_{i j}\right|$. The contact forces are given by

$$
\mathbf{F}_{i j}^{c}= \begin{cases}F_{0} \mathbf{n}_{i j} & \text { if }\left|\mathbf{h}_{i j}\right| \leqslant 2 \epsilon \\ 0 & \text { if }\left|\mathbf{h}_{i j}\right|>2 \epsilon,\end{cases}
$$

where $F_{0}$ denotes the amplitude of the repulsive force. This repulsive force was chosen for its simplicity. It is large enough so that particles do not overlap. If $\left|\mathbf{h}_{i j}\right| \leqslant 2 \epsilon$, the particles are instantaneously pushed away from each other by the repulsive force which is much larger than any hydrodynamic force. During contact, the typical variation of the distance between particles is $O\left(10^{-6} d\right)$. Other, more sophisticated definitions of the repulsive force (such as Hertzian contacts or the exponentially decaying potential used in Stokesian dynamics ${ }^{10}$ ) were also tested and provide very similar particle trajectories. The equations presented above are solved for the velocities of the particles given their spatial positions. The positions are updated in time using a fourth-order Runge-Kutta method with a time step that ensures a displacement of less than $10^{-5} d$ at each time step.

Figure 2(a) shows the initial position of the particles and the corresponding relative trajectories recorded over 10 cycles of shear. Arrows indicate the direction of motion of the particles. The interesting feature is that during the backward part of the first cycle, particles are forced away from their reversible path: particles follow different trajectories, ending further apart than they were initially. This irreversible behavior occurs only during the first cycle. Over the next nine cycles, particles exhibit a reversible behavior, always retracing the same trajectory on the forward and backward portions of the cycles. This behavior is illustrated by the spatio-temporal plot shown in Figure 2(b). This spatio-temporal plot was constructed from the pixels highlighted by the dashed line on Figure 2(a), which was taken from each successive image and plotted side by side. During the first cycle, the spatio-temporal pattern changes indicating irreversibility. After that, reversibility is observed clearly as the patterns repeat.

The irreversible motion of the particles observed here is reminiscent of the behavior observed in homogeneous suspensions ${ }^{5,6,16}$ or for clouds of particles subjected to a periodic flow. ${ }^{7}$ To probe whether solid-solid contacts between particles are responsible for this behavior, the experiment
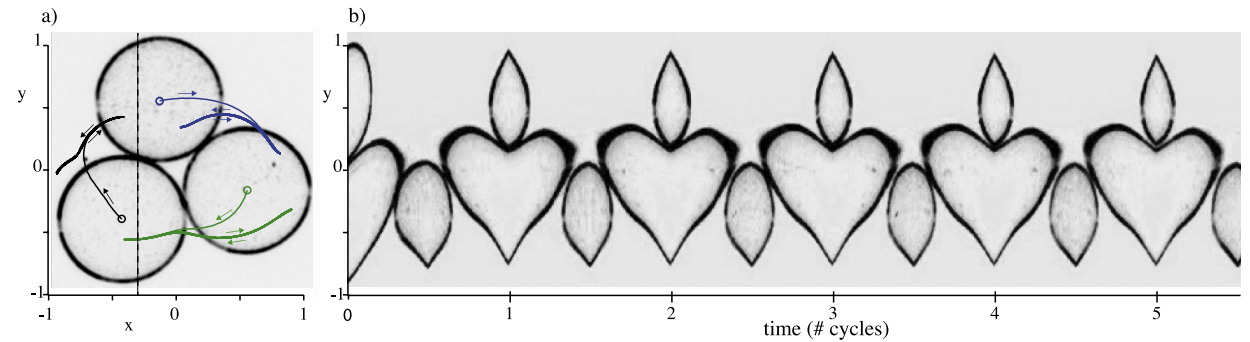

FIG. 2. Experiments: (a) Initial configuration of the cloud of particles and relative trajectories over 10 cycles for particles of Batch III. Arrows indicate the direction of motion of the particles. (b) Spatio-temporal plot of the dashed line shown in (a). A movie is available with the online version of the paper. (Multimedia view) [URL: http://dx.doi.org/10.1063/1.4919728.1] 

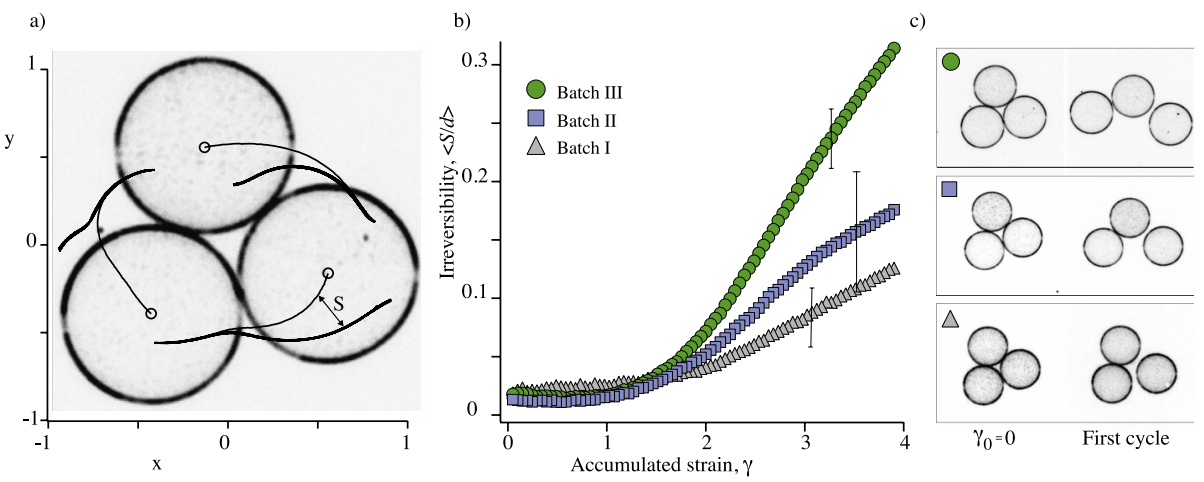

FIG. 3. Experiments: (a) Example of trajectories for particles of Batch III. (b) Magnitude of the irreversibility, $\langle S / d\rangle$, during the first cycle of shear versus accumulated strain for the three batches of particles. Errors bars correspond to the standard deviation calculated over the five experimental runs performed for each roughness. (c) Image of the particles at $\gamma_{0}=0$ and at the end of the first cycle.

was repeated with the three batches of particles having different roughnesses. For each run, the amount of irreversibility, $\langle S / d\rangle$, was quantified as the average separation distance between the forward and backward motions of the first cycle as shown in Figure 3(a). The striking result, shown in Figure 3(b), is that the amplitude of the irreversibility increases as the particle roughness is increased. For larger roughness, the particles end up further apart at the end of first cycle as shown in Figure 3(c). This measurement provides direct evidence that particle solid-solid contacts are at the origin of the irreversible behavior observed in sheared suspensions.

In the following, we investigate if the minimal model presented above can capture the particle dynamics. We assume the particles are initially at contact, forming an equilateral triangle. This defines the particle diameter, $d$, taken as the average initial separation distance between the particles. The model then only contains two adjustable parameters: the magnitude of force, $F_{0}$, and the surface roughness, $\epsilon$. The optimum values for these parameters are determined by minimizing the distance, $\ell=\frac{1}{N} \sqrt{\sum_{i=1}^{t} \sum_{i=1}^{N}\left(\left(x_{i}^{A}-x_{i}^{B}\right)^{2}+\left(y_{i}^{A}-y_{i}^{B}\right)^{2}\right)}$, where A and $\mathrm{B}$ indicate the experimental and simulation data, respectively.

The inset of Figure 4(b) shows $\ell$, as obtained for incremental values of the magnitude of the force, $F_{0}$, and of the particle roughness, $\epsilon$. The distance between simulations and experiments

$\circ$ Experiments

a) - Simulations

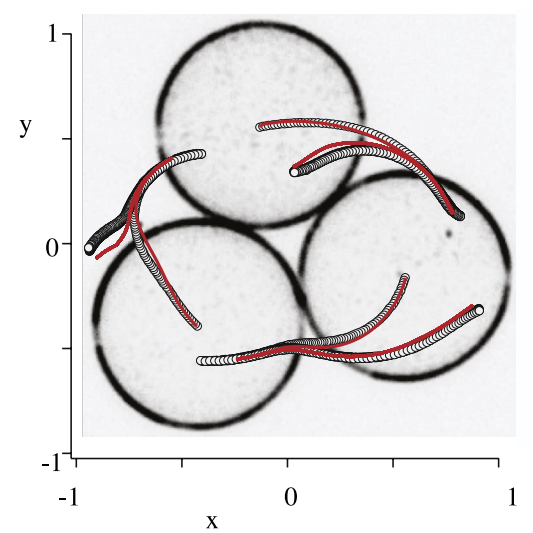

b)

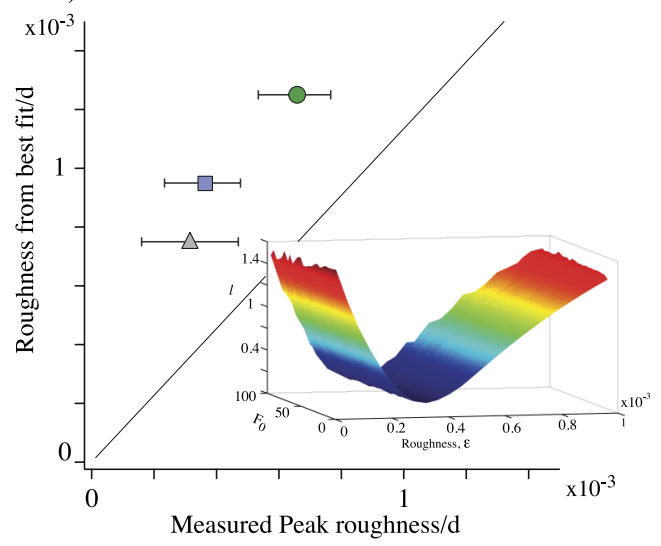

FIG. 4. Experiments and simulations: (a) Comparison of the trajectories obtained from simulations to experimental trajectories for particles of Batch III. (b) Dimensionless roughness obtained from the simulations trajectories providing the best fit with the experimental trajectories versus the dimensionless peak roughness obtained from AFM. Inset: Separation distance, $\ell$, vs. magnitude of the repulsive force, $F_{0}$, and roughness, $\epsilon$. 
behaves as a flat valley suggesting that the magnitude of the force, if chosen in a reasonable range, does not affect significantly the particle trajectories. Conversely, changing the roughness provides a well-defined minimum separation distance, which defines a roughness that optimizes the agreement between experimental and simulation trajectories. Trajectories obtained with the optimum roughness are shown in Figure 4(a): as observed in the experiment, the first cycle is irreversible, while the following cycles are reversible. This minimal model, which only includes a solid-solid contact force and the normal component of the lubrication force, quantitatively reproduces the trajectories that were measured experimentally.

In Figure 4(b), the roughness AFM measurements are compared to the optimum roughness obtained from the best fit of the numerical model to the experimentally measured trajectories. The results are close (within a factor 2) to the AFM peak values; the measured RMS values are one order of magnitude smaller than those obtained from the minimization, see Figure 1(b). This suggests that the relevant roughness is given by the peak value. This is consistent since when particles approach, the minimal separation distance at which the particles can touch is given by the peak value of the roughness, not by the RMS.

We have reported on highly resolved experimental measurements of the individual trajectories of small number of particles subjected to a periodic shear under creeping-flow conditions. These experiments provide evidence that particles experience solid-solid contacts, as increasing the roughness was found to increase the irreversibility of the particle trajectories. A minimal model that includes only hydrodynamic drag, the normal component of the lubrication interaction, and contact forces provides a quantitatively accurate description of the particle dynamics and consequently supports the idea that particle contacts are responsible for the experimental observations.

The authors thank P. Cervetti, S. Noël, and F. Ratouchniack for machining and helping with the design of the setup and A. Rangis (Aix-Marseille University) for the AFM measurements. This work was supported by the National Science Foundation (Grant No. 0968313), ANR JCJC SIMI 9 and has been carried out in the framework of the Labex MEC (ANR-10-LABX-0092) and of the A*MIDEX project (ANR-11-IDEX-0001-02), funded by the "Investissements d'Avenir" French Government program managed by the French National Research Agency (ANR).

${ }^{1}$ P. A. Arp and S. G. Mason, "The kinetics of flowing dispersions IX. Doublets of rigid spheres (Experimental)," J. Colloid Interface Sci. 61, 44 (1977).

${ }^{2}$ I. Rampall, J. R. Smart, and D. T. Leighton, "The influence of surface roughness on the particle-pair distribution function of dilute suspensions of non-colloidal spheres in simple shear flow," J. Fluid Mech. 339, 1 (1997).

${ }^{3}$ M. S. Ingber, A. A. Mammoli, P. Vorobieff, T. McCollam, and A. L. Graham, "Experimental and numerical analysis of irreversibilities among particles suspended in a Couette device," J. Rheol. 50, 99 (2006).

${ }^{4}$ F. Blanc, F. Peters, and E. Lemaire, "Experimental signature of the pair-trajectories of rough spheres in the shear-induced microstructure in non-colloidal suspensions," Phys. Rev. Lett. 107, 208302 (2011).

${ }^{5}$ B. Metzger and J. E. Butler, "Irreversibility and chaos: Role of long range hydrodynamic interactions in sheared suspensions," Phys. Rev. E 82, 051406 (2010).

${ }^{6}$ B. Metzger, P. Pham, and J. E. Butler, "Irreversibility and chaos: Role of lubrication interactions in sheared suspensions," Phys. Rev. E 87, 052304 (2013).

${ }^{7}$ B. Metzger and J. E. Butler, "Clouds of particles in a periodic shear flow," Phys. Fluids 24, 021703 (2012).

${ }^{8}$ E. Lerner, G. Düring, and M. Wyart, "Simulations of driven overdamped frictionless hard spheres," Comput. Phys. Commun. 184(3), 628-637 (2013).

${ }^{9}$ R. Seto, R. Mari, J. F. Morris, and M. M. Denn, "Discontinuous shear thickening of frictional hard-sphere suspensions," Phys. Rev. Lett. 111, 218301 (2013)

${ }^{10}$ G. Bossis and J. F. Brady, "Dynamic simulation of sheared suspensions. I. General method,” J. Chem. Phys. 80, 5141 (1984),

${ }^{11}$ M. Abbas, E. Climent, and O. Simonin, "Fully coupled simulations of non-colloidal monodisperse sheared suspensions," Chem. Eng. Res. Des. 85, 778 (2007).

${ }^{12}$ K. Yeo and M. R. Maxey, "Simulation of concentrated suspensions using the force-coupling method," J. Comput. Phys. 229, 2401 (2010).

${ }^{13}$ F. R. da Cunha and E. J. Hinch, "Shear-induced dispersion in a dilute suspension of rough spheres," J. Fluid Mech. 309, 211 (1996).

${ }^{14}$ D. Nečas and P. Klapetek, "Gwyddion: An open-source software for SPM data analysis," Cent. Eur. J. Phys. 10(1), 181 (2012).

${ }^{15}$ G. K. Batchelor and J. T. Green, "The hydrodynamic interaction of two small freely-moving spheres in a linear flow field," J. Fluid Mech. 56, 401 (1972).

${ }^{16}$ D. J. Pine, J. P. Gollub, J. F. Brady, and A. M. Leshansky, "Chaos and threshold for irreversibility in sheared suspensions," Nature 438, 997 (2005). 